\title{
Pathway activity inference for multiclass disease classification through a mathematical programming optimisation framework
}

\author{
Lingjian Yang ${ }^{1}$, Chrysanthi Ainali², Sophia Tsoka ${ }^{2 *}$ and Lazaros G Papageorgiou ${ }^{1 *}$
}

\begin{abstract}
Background: Applying machine learning methods on microarray gene expression profiles for disease classification problems is a popular method to derive biomarkers, i.e. sets of genes that can predict disease state or outcome. Traditional approaches where expression of genes were treated independently suffer from low prediction accuracy and difficulty of biological interpretation. Current research efforts focus on integrating information on protein interactions through biochemical pathway datasets with expression profiles to propose pathway-based classifiers that can enhance disease diagnosis and prognosis. As most of the pathway activity inference methods in literature are either unsupervised or applied on two-class datasets, there is good scope to address such limitations by proposing novel methodologies.

Results: A supervised multiclass pathway activity inference method using optimisation techniques is reported. For each pathway expression dataset, patterns of its constituent genes are summarised into one composite feature, termed pathway activity, and a novel mathematical programming model is proposed to infer this feature as a weighted linear summation of expression of its constituent genes. Gene weights are determined by the optimisation model, in a way that the resulting pathway activity has the optimal discriminative power with regards to disease phenotypes. Classification is then performed on the resulting low-dimensional pathway activity profile.

Conclusions: The model was evaluated through a variety of published gene expression profiles that cover different types of disease. We show that not only does it improve classification accuracy, but it can also perform well in multiclass disease datasets, a limitation of other approaches from the literature. Desirable features of the model include the ability to control the maximum number of genes that may participate in determining pathway activity, which may be pre-specified by the user. Overall, this work highlights the potential of building pathway-based multi-phenotype classifiers for accurate disease diagnosis and prognosis problems.
\end{abstract}

Keywords: Disease classification, Microarray, Pathway activity, Mathematical programming, Optimisation

\section{Background}

The popularity of microarray technology as means of deriving a comprehensive view of gene expression under particular environmental stimuli has necessitated the development of computational strategies for linking expression patterns to sample phenotypes [1,2]. In charactering disease, the gene expression matrix serves as input to a

\footnotetext{
*Correspondence: Sophia.tsoka@kcl.ac.uk; l.papageorgiou@ucl.ac.uk ${ }^{2}$ Department of Informatics, School of Natural and Mathematical Sciences, King's College London, London WC2R 2LS, UK

'Centre for Process Systems Engineering, Department of Chemical Engineering, University College London, London WC1E 7JE, UK
}

classification task where each sample is allocated to a relevant phenotypic class via specific gene signatures or biomarkers that can best differentiate between outcomes. Such disease classification tasks have been successful in deriving biomarkers for diagnosis [3], prognosis [4-7] and response to treatment $[8,9]$ in complex disorders.

Despite successful reports, disease classification is impeded by the so-called "large $\mathrm{p}$ small n" property, whereby the number of samples (or instances) is typically several orders of magnitude smaller than the number of genes (or features), making it difficult to extract reliable information from transcriptomic profiles [10]. Feature reduction 
methods are therefore employed as means of extracting a smaller set of genes, able to discriminate between disease states. We note as example methods based on partial least squares [11], heuristic breadth-first search algorithm [12], and ensemble feature selection methods [13]. Subsequently, a classifier can be trained on the reduced feature set to predict the disease status or prognostic characteristic of any given samples [14-17].

Such diagnostic or prognostic profiles relate to genes that do not act in isolation, but in fact work in concert, forming sub-networks that collectively modulate or determine cell fate. Accounting for such molecular synergies in feature reduction and disease classification protocols can also alleviate challenges of single-gene classifiers related to cellular heterogeneity in tissue, genetic heterogeneity among patients, measurement noise [18-20], thereby leading to increased biological interpretability of biomarkers and enhancing insights into the mechanisms of the disease [21-23]. Therefore, feature selection and classification methods where all genes are treated independently are increasingly replaced by approaches where the effects of groups of genes on disease prediction are considered simultaneously. Such gene sets can either reflect curated biochemical pathways or functional modules derived from protein interaction networks [19,24-34].

The availability of pathway information from publc databases, for example Kyoto Encyclopedia of Genes and Genomes (KEGG) [35], Gene Ontology (GO) [36] and Reactome [37], provide the possibility of analysing functional sets of genes that fall within common pathways and identifying the disease-relevant pathways as biomarkers. Initial efforts of gene-set based approaches included gene set enrichment analysis [38], which calculates to what extent a set of genes show statistically significant difference between samples belonging to either of the two phenotypes. Other similar computational tools have also been reported [39-44]. However, those statistical frameworks commonly assign one score for each set of genes to quantify the deregulation of this gene-set under disease status of interest, but do not provide more information on the level of gene-set deregulation for each sample. It is argued that this drawback compromises their potential in personalised pathway analysis [26].

Therefore, a more informative approach may be to assign a score to each pathway and sample, which represents the activity of that particular pathway for that sample [19,25-28,45-47]. The mean and median expression value across all constituent genes within a pathway, termed pathway activity, has been proposed in [28]. Other studies produce pathway activity measures based on principle component analysis (PCA) to derive the top principle component that captures the maximum variance in the dataset $[26,45,48,49]$. More recently a supervised greedy search algorithm was proposed that ranks genes according to their individual discriminative power and then searches for a subset of highly ranked genes whose averaged expression profiles yield better discriminative power [19]. This method was modified so that it accounts for up- and down-regulated genes by assigning positive sign and negative sign respectively [27]. Both methods are inherently applicable to binary classification problems. A statistical inference method [25] proposes to aggregate the probabilistic evidence of all genes within a pathway for predicting a sample into one of the two phenotypes. Other relevant studies based on the concept of pathway activity either require other biological information as prior, for example copy number variation and protein interactions $[20,47,50]$ or are not designed for classification tasks $[47,51]$.

Pathway activity-based classification approaches provide competitive or higher prediction accuracy when compared to traditional single genes-based classifiers [19,52], so extending or refining their use is a promising avenue for biomarker discovery. Despite rapidly increasing interest in developing novel and robust pathway activity inference methods, most of the existing methods still use rather simple means of summarising the expression patterns of either some or all constituent genes into the composite pathway level attribute, for example the mean or median value of sample expression across all or a subset of constituent genes [19,28]. PCA-based methods [26,45,48,49] calculate the first principal component, representing the maximum variance of the data set, as pathway activity. However such methods do not take into account the phenotype information of samples. Furthermore, some current pathway activity inference methods are constrained to two-phenotype (binary) classification problems $[19,20,25,27]$, disallowing their use in more complex problems of multi-phenotype classification.

In this work, we propose a novel multiclass method that infers pathway activity in a supervised manner. The proposed method summarises expression patterns of constituent genes into pathway activity via weighted linear summation of gene expression. As opposed to some methods in literature where gene weights are taken as a prior, in our work gene weights are decided by the model, so that the constructed pathway activity can optimally distinguish samples from different phenotypes. Furthermore, the mathematical framework of this method offers the ability to the user to explicitly constraint the maximum number of constituent genes contributing to pathway activity inference. Using a number of published gene expression profile datasets, we show that this pathway activity inference method is robust in terms of the number of constituent genes allowed to determine the pathway activity metric. Comparative analyses show that the method is an effective means of reducing classification features, as it either outperforms or at least matches competing pathway activity inference methods in two-phenotype 
disease classification problems, and provides significantly better classification rates in multi-phenotype classification problems.

\section{Methods}

Data sources

Complex diseases such as breast cancer and psoriasis are the product of multiple gene interactions that collectively contribute to the etiology of the disease through largely unknown mechanisms [53]. Breast cancer is the most frequently diagnosed malignancy and has been intensively studied by gene expression profiling [4-6,54-57]. Psoriasis is a systemic, inflammatory skin disease with autoimmune underpinnings affecting 2-3\% of the world population [58-60]. Prostate tumor is the most frequently diagnosed cancer in American men [61] and displays a broad range of clinical and histological behaviors [3,62]. Diffuse large B-cell lymphoma (DLBCL) is the most common lymphoid malignancy in adults [63] with less than $40 \%$ patients responding desirably to the current therapy while the remainders succumb to the disease, highlighting the unidentified molecular heterogeneity in the tumors [64].

A total number of 8 published microarray gene expression profiles were obtained that represent these diseases (Table 1). In terms of disease phenotypes in these datasets, used as class outcomes in the relevant classification tasks, for psoriasis samples are either lesional or nonlesional tissue from psoriasis patients, as well as healthy controls $[65,66]$. For breast cancer, 49 samples belong to three disease classes, apocrine, basal and luminal [67]; 139 samples are divided into healthy, luminal, ERBB2 and basal [68]; expression profiles of 230 breast cancer patients 48 of whom became residual invasive cancer free in the breast or lymph nodes after a 6-month preoperative chemotherapy and the remainder still had residual invasive cancer after the treatment. Gene expression data were generated using specimens of breast cancer before any treatment [8]; lymph-node negative breast cancer patients with some of them diagnosed with distant metastasis [4]. For prostate cancer, 102 expression profiles are used to distinguish tumour samples from normal samples [3]. Finally, 77 expression profiles of patients either diagnosed with diffuse large B-cell lymphoma or follicular lymphoma (FL) are used [63].

All microarray datasets have been obtained on Affymetrix platforms. For each dataset, raw data have been downloaded and pre-processed using the Bioconductor package LIMMA [69]. KEGG C2 functional gene sets have been downloaded from MsigDB database (v3.0, Sep 2010) [70], which included a total number of 186 curated pathways and 5267 genes.

Table 1 Datasets

\begin{tabular}{|c|c|c|c|c|}
\hline Dataset & Disease & Samples & Samples per phenotype & Source \\
\hline \multirow[t]{3}{*}{ Swindell [65] } & Psoriasis & 180 & Healthy control: 64; & GSE13355 \\
\hline & & & Psoriatic non-lesional skin: 58; & \\
\hline & & & Psoriatic lesional skin: 58 & \\
\hline \multirow[t]{3}{*}{ Yao [66] } & Psoriasis & 82 & Healthy control: 21; & GSE14905 \\
\hline & & & Psoriatic non-lesional skin: 28; & \\
\hline & & & Psoriatic lesional skin: 33 & \\
\hline \multirow[t]{3}{*}{ Farmer [67] } & Breast cancer & 49 & Apocrine tumour: 6 & GSE1561 \\
\hline & & & Basal tumour: 16; & \\
\hline & & & Luminal tumour: 27 & \\
\hline \multirow[t]{4}{*}{ Pawitan [68] } & Breast cancer & 139 & Normal: 37; & GSE1456 \\
\hline & & & Luminal tumour: 62; & \\
\hline & & & ERBB2: 15; & \\
\hline & & & Basal: 25 & \\
\hline \multirow[t]{2}{*}{ Singh [3] } & Prostate cancer & 102 & Normal: 50; & www.broadinstitute.org \\
\hline & & & Tumour: 52 & \\
\hline \multirow[t]{2}{*}{ Shipp [63] } & DLBCL & 77 & DLBCL: 58; & www.broadinstitute.org \\
\hline & & & Follicular lymphoma: 19 & \\
\hline \multirow[t]{2}{*}{ Popovici [8] } & Breast cancer & 230 & Residual invasive cancer: 182 & GSE24061 \\
\hline & & & No residual invasive cancer: 48 & \\
\hline \multirow[t]{2}{*}{ Desmedt [4] } & Breast cancer & 198 & Metastatic: 51; & GSE7390 \\
\hline & & & Non-metastatic: 147 & \\
\hline
\end{tabular}




\section{Pathway activity-based classification procedure}

An overview of the computational procedure developed for pathway activity-based disease classification is illustrated in Figure 1. A microarray gene expression profile and a set of pathways with their constituent genes form the input to create pathway-specific gene expression matrices. For each pathway, $m$ denotes member genes, $s$ samples and $A_{s m}$ the expression value of gene $m$ in sample s. $A_{s m}$ are standardised to $G_{s m}$ by subtracting the population mean from the raw value and then dividing by the standard deviation. The first stage of our computational procedure derives a new composite feature, pathway activity $p a_{s}$, from the standardised pathway specific gene expression profile $G_{s m}$. In the second stage of our protocol, the inferred pathway activities for all pathways are assembled to form a pathway activity profile matrix, on where a classifier is trained to predict the phenotype of a new sample. In next section, we present a novel mathematical model, which infers pathway activity with optimal classification accuracy.

\section{A novel mathematical programming formulation to infer pathway activity}

The indices, parameters and variables used in the model to infer pathway activity for each pathway are described here and are listed in Additional file 1. Two sets of positive variables $r p_{m}$ and $r n_{m}$ are introduced, quantifying the positive and negative weights of gene $m$ towards pathway activity inference. For sample $s$, pathway activity, $p a_{s}$, is defined as the summation of the standardised gene expression values, $G_{s m}$ multiplied by the gene weight $\left(r p_{m}-r n_{m}\right)$ over all member genes:

$$
p a_{s}=\sum_{m=1}^{M} G_{s m}\left(r p_{m}-r n_{m}\right) \quad \forall s=1,2, \ldots S
$$

where $M$ is the total number of member genes for this particular pathway and $S$ is the total number of samples. Both positive and negative weights of a gene $m$ are defined as positive continuous variables; their values are determined by the optimisation model. One set of binary




variables, $L_{m}$, which takes values of either 0 or 1 has been introduced, while equations (2) and (3) below ensure that for each gene $m$ at most one of $r p_{m}$ and $r n_{m}$ can take positive values:

$$
\begin{array}{ll}
r p_{m} \leq L_{m} & \forall m=1,2, \ldots, M \\
r n_{m} \leq 1-L_{m} & \forall m=1,2, \ldots, M
\end{array}
$$

When $L_{m}=1, r p_{m}$ can take any value between 0 and 1 while $r n_{m}$ is forced to be equal to 0 ; otherwise when $L_{m}=$ $0, r p_{m}$ is forced to be equal to 0 while $r n_{m}$ can be between 0 and 1. In either case, both $r p_{m}$ and $r n_{m}$ can be equal to 0 , which means this particular gene has zero weight in inferring pathway activity. Overall, a gene can have positive, negative or zero weight towards the composite feature construction. For normalisation purpose, the summation of absolute gene weights should be equal to one:

$$
\sum_{m=1}^{M}\left(r p_{m}+r n_{m}\right)=1
$$

Inspired by Lee et al. [19], where a small subset of member genes is selected (usually less than 7) to construct pathway activity, we add constraints to limit the number of genes having non-zero weights in inferring pathway activity. Thus a new set of binary variables, $W_{m}$, are introduced to the model to indicate whether a member gene $m$ is active, i.e. having non-zero weights in constructing pathway activity or not:

$$
r p_{m}+r n_{m} \leq W_{m} \quad \forall m=1,2, \ldots, M
$$

If $W_{m}$ takes the value of 0 then both positive weight $\left(r p_{m}\right)$ and negative weight $\left(r n_{m}\right)$ of gene $m$ are forced to be equal to 0 , while when $W_{m}$ is equal to 1 , gene $m$ is allowed to take any weight $\left(r p_{m}-r n_{m}\right)$ between -1 and 1 . The next equation restricts the maximum number of genes allowed to have non-zero weights to a manually specified value $(\mathrm{NoG})$ :

$$
\sum_{m=1}^{M} W_{m} \leq N o G
$$

In the case where $N o G$ is equal to or larger than the number of member genes available in the pathway, the constraint is redundant as all the member genes will be allowed to take any weight $\left(r p_{m}-r n_{m}\right)$ between -1 and 1 . We aim to construct pathway activity as a feature with good discriminative power, which can separate samples from different phenotypes as much as possible.

For each phenotype/class $c$, two continuous variables have been introduced as $L O_{c}$ and $U P_{c}$, denoting the lower and upper bound respectively, of the range of pathway activity for phenotype $c$. In addition, a set of binary variables, $E_{s}$, have been introduced, defined by: together with the following constraints:

$$
\begin{array}{lr}
0 \leq p a_{s}-L O_{c}+U\left(1-E_{s}\right) & \forall s=1,2, \ldots, S, c_{s} \\
\mathrm{pa}_{s}-U P_{c}-U\left(1-E_{s}\right) \leq 0 & \forall s=1,2, \ldots, S, c_{s}
\end{array}
$$

where $c_{s}$ is the phenotype for sample $s$ and $U$ is an arbitrarily large positive number. On the constructed pathway activity, ranges of different classes are not allowed to overlap. A set of binary variables, $Y_{k c}$, have been introduced as follows:

$Y_{k c}=\left\{\begin{array}{l}1, \text { if upper bound of range for class } \mathrm{k} \text { is lower than } \\ \text { lower bound of range } \\ \text { for class c on pathway activity; } \\ 0, \text { otherwise }\end{array}\right.$

The additional two sets of constraints have been introduced to guarantee the non-overlapping condition:

$$
\begin{array}{lc}
U P_{k}+\varepsilon \leq L O_{c}+U\left(1-Y_{k c}\right) & \forall k<c \\
U P_{c}+\varepsilon \leq L O_{k}+U Y_{k c} & \forall k<c
\end{array}
$$

where $\varepsilon$ is an arbitrarily small positive number ensuring that pair-wise classes do not share a border. Equations (9) and (10) are generated for each pair of classes. The objective of the optimisation problem is to infer the pathway activity such that it is as discriminative as possible, i.e. as many samples as possible can fall within the range of its corresponding classes $\left(E_{s}=1\right)$. In other words, the objective function is to minimise the number of misclassified samples:

$$
\min \sum_{s=1}^{S}\left(1-E_{s}\right)
$$

The resulting mathematical programming-based formulation for inferring pathway activity is summarised below:

Objective function (11)

Subject to:

Pathway activity definition (1)

Positive and negative gene effect constraints (2) and (3)

Normalisation constraint (4)

Restriction of the number of active genes (5) (6)

Pathway activity enclosing constraints (7) and (8)

Non-overlapping constraints for ranges of different classes (9) and (10)

$L_{m}, E_{s}, W_{m}, Y_{k c} \in\{0,1\} ; r p_{m}, r n_{m} \geq 0 ; p a_{s}, L O_{c}, U P_{c}$ : unrestricted

$$
E_{s}=\left\{\begin{array}{l}
1, \text { if activity value of sample s falls within the lower and upper bounds of } \\
\text { its class range; } \\
0, \text { otherwise }
\end{array}\right.
$$


The proposed mathematical programming formulation consists of a linear objective function and a number of linear constraints. The linearity and presence of binary and continuous variables define a mixed integer linear programming (MILP) model, named DIGS (DIfferential Gene Signatures) here, and can be solved to global optimality using some of the standard algorithms like branchand-bound.

To facilitate the use of DIGS, we make available the GAMS executable, example input files and user guide at www.ucl.ac.uk/ uceclap/DIGS.

\section{Comparison of the DIGS model with genes-based methods} and other pathway activity inference methods

To compare the results obtained with the DIGS model, we have implemented a number of pathway activity methods from the literature (summarised in Table 2). In overview, these methods include: i) the method that uses the microarray gene expression profile without pathway information, for example SG; ii) the method that utilises pathway information but is based on the pathway specific gene expression profile instead of inferring pathway activity, for example per_pathway, and iii) those that take advantage of pathway information and infer pathway activity, for example [19,28,45].

In detail, comparative results are presented by implementation of the following methods: i) a genes-based approach has been implemented for comparison where, given a whole gene expression profile, a feature selection [71] method is applied to select a subset of top genes with the best discriminative power for classification. The multiclass feature selection method [71] used here employs a distance metric, for example weighted $L_{1}$ metric or K-L divergence and gives a subset of top attributes/ genes with respect to the aggregated pair-wise class distances, where the number of attributes in the subset obtained is pre-set by the user. A classifier is then trained using only the small subset of discriminative genes for disease classification problems; ii) the Ainali et al. [72] method, where each pathway-specific gene expression profile is treated independently, i.e. training and testing are conducted for each pathway-specific expression profile separately and classification accuracies across all pathways are averaged to obtain the final classification rate (referred

Table 2 Overview of Evaluated Methods

\begin{tabular}{|c|c|}
\hline \multirow[t]{3}{*}{ Guo et al. [28] } & Abbreviation: Mean \\
\hline & Computational basis: Pathway activity \\
\hline & $\begin{array}{l}\text { Description: Create pathway-specific gene expression profiles; for each pathway, pathway activity for sample is its mean } \\
\text { expression value among all member genes; a classifier is trained on pathway activity profile. }\end{array}$ \\
\hline \multirow[t]{3}{*}{ Guo et al. [28] } & Abbreviation: Median \\
\hline & Computational basis: Pathway activity \\
\hline & $\begin{array}{l}\text { Description: Create pathway-specific gene expression profiles; for each pathway, pathway activity for sample is its median } \\
\text { expression value among all member genes; a classifier is trained on pathway activity profile. }\end{array}$ \\
\hline \multirow[t]{3}{*}{ Bild et al. [45] } & Abbreviation: PCA \\
\hline & Computational basis: Pathway activity \\
\hline & $\begin{array}{l}\text { Description: Create pathway-specific gene expression profiles; for each pathway, top principal component is } \\
\text { calculated as the pathway activity; a classifier is trained on pathway activity profile. }\end{array}$ \\
\hline \multirow[t]{3}{*}{ Lee et al. [19] } & Abbreviation: CORGS \\
\hline & Computational basis: Pathway activity \\
\hline & $\begin{array}{l}\text { Description: Create pathway-specific gene expression profiles; for each pathway, apply t-test to rank genes and } \\
\text { perform a greedy search to find a subset of genes whose averaged expression values is locally maximal in } t \text {-test } \\
\text { value; a classifier is trained on pathway activity profile; only applicable for two-class problems. }\end{array}$ \\
\hline \multirow[t]{3}{*}{ Ainali et al. [72] } & Abbreviation: Per_pathway \\
\hline & Computational basis: Single genes \\
\hline & $\begin{array}{l}\text { Description: Create pathway-specific gene expression profiles; a classifier is trained on each pathway-specific gene expression } \\
\text { profile separately, and prediction rates achieved by all pathway classifiers are averaged as the final prediction rate. }\end{array}$ \\
\hline \multirow[t]{3}{*}{ Single Genes } & Abbreviation: SG \\
\hline & Computational basis: Single genes \\
\hline & Description: Apply [71] to select a subset of top genes; a classifier is trained on reduced gene expression profile \\
\hline \multirow[t]{3}{*}{ Proposed in this work } & Abbreviation: DIGS \\
\hline & Computational basis: Pathway activity \\
\hline & $\begin{array}{l}\text { Description: Create pathway-specific gene expression profiles; Apply the proposed DIGS model to construct pathway activity } \\
\text { as weighted linear summation of gene expressions; a classifier is trained on pathway activity profile. }\end{array}$ \\
\hline
\end{tabular}


as per_pathway), and iii) the two methods from Guo et al. [28] (referred as mean and median, respectively), which take either the mean or median gene expression values of all genes within a pathway for each sample. The Bild et al. [45] approach (referred as $P C A$ ) of using the first principal component as representation of pathway activity, which represents a family of principal component analysis-based methods [26,48,49]. The Lee et al. [19] method, which identifies and averages a subset of condition-responsive genes (referred as CORGs), which has been implemented only for two-phenotype disease classification problems, as it is not suited to multi-class problems.

\section{Evaluation of classification performance}

The performance of the various pathway activity metrics is evaluated by the classification accuracy achieved across the eight disease datasets. For each dataset, samples are split randomly in training and testing sets of 70 and $30 \%$ respectively and this procedure is repeated fifty times. Composite features are constructed using Mean, Median, CORGs, PCA and DIGS on the training samples, resulting in low dimensionality matrix of samples across pathway activities, on which five popular classifiers SMO [73], Neural Network (NN) [74], K-Nearest-Neighbours (K-NN) [75], Logistic Regression (Logistic) [76] and Hyperbox (HB) [77] are trained. The classifiers are then tested on the testing sample set and the prediction accuracy is calculated as the number of correctly classified samples divided by the total number of testing samples, averaged across the fifty training/testing sets.

The above procedure is modified where pathway activities are not used, i.e. in the SG and per_pathway approaches. In the genes-based approach, the feature selection method [71] has been applied using training samples only and the top genes are selected. The number of top genes is set to be identical to the number of pathways (i.e. 186) in order to derive comparable dimensionalities between the pathway activity-based and the genes-based approach. For the per_pathway approach, each of the 5 classifiers have been trained using training samples only and then validated on the testing samples sets for each pathway separately.

Overall, 8 microarray gene expression profiles (dataset), 7 competing methods (method) and 5 classifiers (classifier) are employed in our study. For each combination of dataset, method and classifier, classification accuracies over 50 individual testing sets are averaged as the prediction accuracy for this combination. It is important to note that Lee et al. [19] is applicable for only two-phenotype problems, therefore we divide the 8 datasets into a group of 4 binary datasets and the other group of 4 multiclass datasets. For the binary classification comparison, for each method we average the prediction accuracies over all 4 binary datasets and all 5 classifiers, which gives a comprehensive indication of the efficiency of the evaluated methods (i.e. Mean, Median, PCA, CORGs, per_pathway, $\mathrm{SG}$ and the proposed DIGS). For the multiclass case, the same analysis is applied and all comparative analyses are discussed in the next section.

The DIGS model has been implemented in the General Algebraic Modelling System (GAMS) [78] using the CPLEX MILP solver in a CentOS 5.264 bit Unix computer environment. The optimality gap is set as 0 . Computational resource limit is set as 200 seconds per run. Among the 5 classifiers SMO, NN, K-NN and Logistic have been implemented in WEKA machine learning software [79] with the following parameters for $\mathrm{NN}$ : hidden layers 2, learning rate 0.1 , momentum 0.2 , training time 10000; and for $\mathrm{K}-\mathrm{NN}$ : the number of nearest neighbours is selected as 5 . For other classifiers, their default settings have been retained. HB has been reproduced in GAMS according to its original publication [77].

\section{Results and discussion}

In this work, we propose an optimisation-based model that infers a pathway activity metric as a weighted linear combination of the constituent gene expression values. The DIGS model can identify a subset of pathway constituent genes with cardinality no more than the userspecified value, NoG, whose expression can be combined via different weights to best separate samples from different phenotypes. The effect of NoG is illustrated through sensitivity analysis below, followed by a comparison of the model against a variety of disease classification strategies, including both single-gene and pathway activity based approaches.

\section{Sensitivity analysis for NoG, influencing the number of active genes in constructing pathway activity}

Parameter NoG determines the maximum number of pathway member genes that have non-zero weight in activity inference. Tuning this parameter is important as a small value may not fully utilise the discriminative member genes, while an excessively large value may potentially cause over-fitting, i.e. in the case where too many genes are allowed to take non-zero weights for pathway activity against a relatively small number of training samples, leading to decreased prediction accuracy.

Here, the DIGS model is applied to infer pathway activity with $N o G$ set to 5, 10,15 and 20, followed by training and testing using a range of classifiers for each microarray dataset. As a comparison, DIGS is also run with NoG set equal to the number of member genes for each pathway, so as to allow all member genes in a pathway to take non-zero weights for pathway activity inference. The prediction rates achieved by these different values of NoG are denoted by DIGS_5, DIGS_10, DIGS_15, DIGS_20 and DIGS_ALL and are shown in Figure 2A and B with SMO and NN classifiers and other classifiers in Additional file 2. 


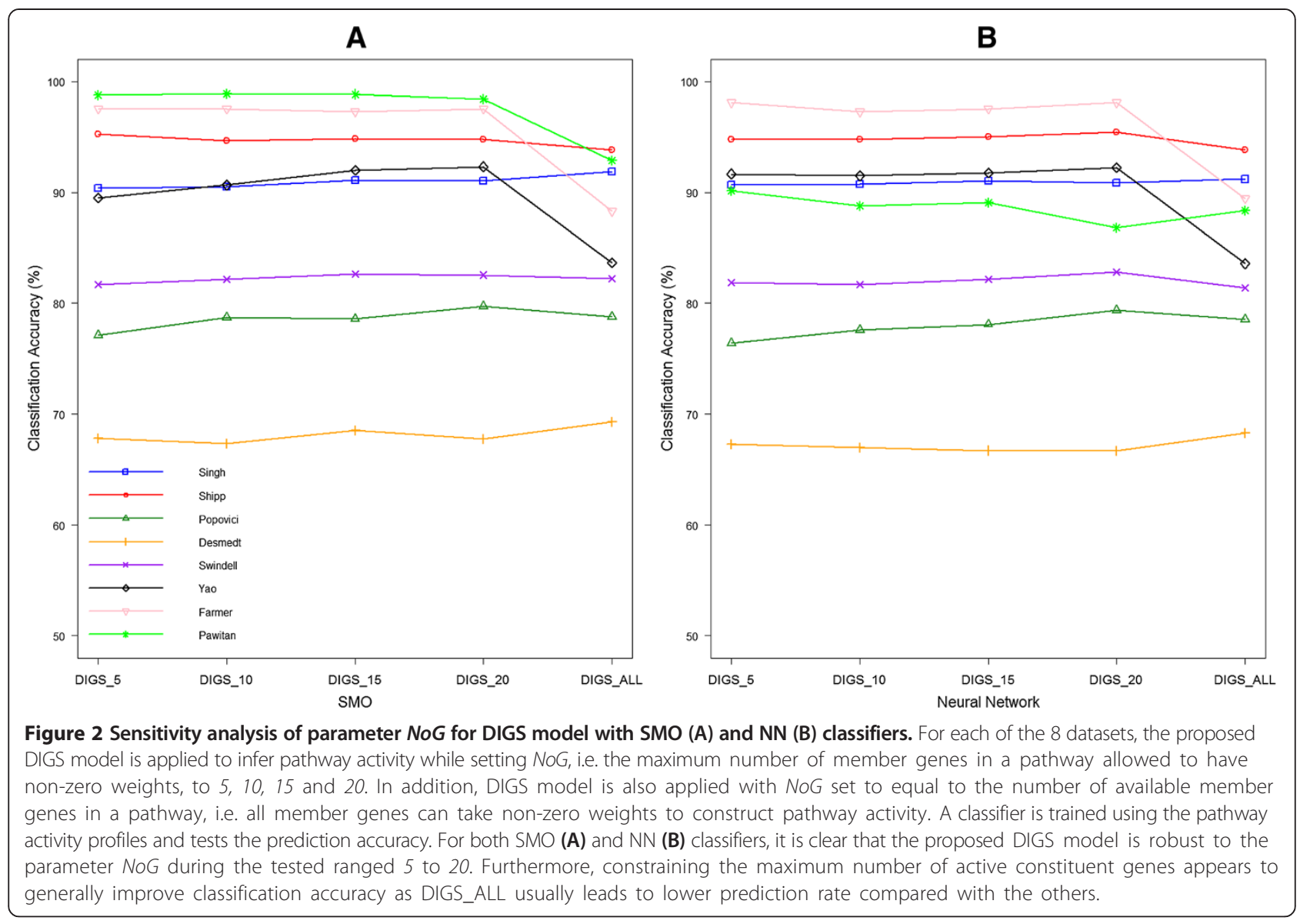

Generally, the DIGS model is robust with respect to parameter $N o G$, as in the range of 5 to 20 , classification prediction performance is found to be mostly stable, with some improvement observed between NoG 5 and 20 . Overall, it is noted that prediction performance is casedependent, not only depending on the dataset under investigation, but also varying with the particular pathway in question (e.g. number of member genes per pathway). In some cases, some improvement is observed against the case of no selection, for example on Yao, Farmer and Pawitan datasets with SMO classifier classification rates increase from $83.7 \%, 88.3 \%$ and $92.9 \%$ to $89.5 \%, 97.6 \%$ and $98.8 \%(\mathrm{NoG}=5)$ respectively (Figure $2 \mathrm{~A})$.

The model performs well even in the case where the number of genes is not reduced (see DIGS_ALL in Figures 2 and Additional file 2), indicating that, although reducing the total number of genes per pathway through parameter NoG may be beneficial to a particular application, it is by no means compulsory. Therefore, NoG offers the flexibility of feature reduction, if looking into the effect of a subset of genes is desired, without imposing any additional limitations that would stem from cases where parameter specification would be mandatory. For the implementations discussed below, NoG equal to a value of ten was chosen as a sensible compromise of the effects discussed above.

\section{Classification rate comparison across other methods}

The performance of the proposed DIGS model against other competing methods in literature is compared and discussed here. As described in the Methods section, extensive comparisons were implemented across 8 datasets (collectively referred to as dataset) and 7 competing methods (method). To also account for the effect of classifier choice in the computational procedure, we tested the DIGS model across 5 classifiers (classifier). The results across all dataset, method and classifier combination are illustrated in Figure $3 \mathrm{~A}$ and B (for 5-NN and Neural Network classifiers) and in the Additional file 3 (for SMO, $\mathrm{HB}$ and logistic).

It is obvious from Figure 3A that using $5-\mathrm{NN}$ as classifier DIGS-based classification approach achieves higher classification rates than other pathway activity inference methods, including Mean, Median, PCA, CORGs. On all 8 datasets, DIGS model inferring pathway activity has always outperformed other pathway activity inference methods. It is not a surprise as DIGS seeks to infer pathway activity as of optimal discriminative power. It is also 


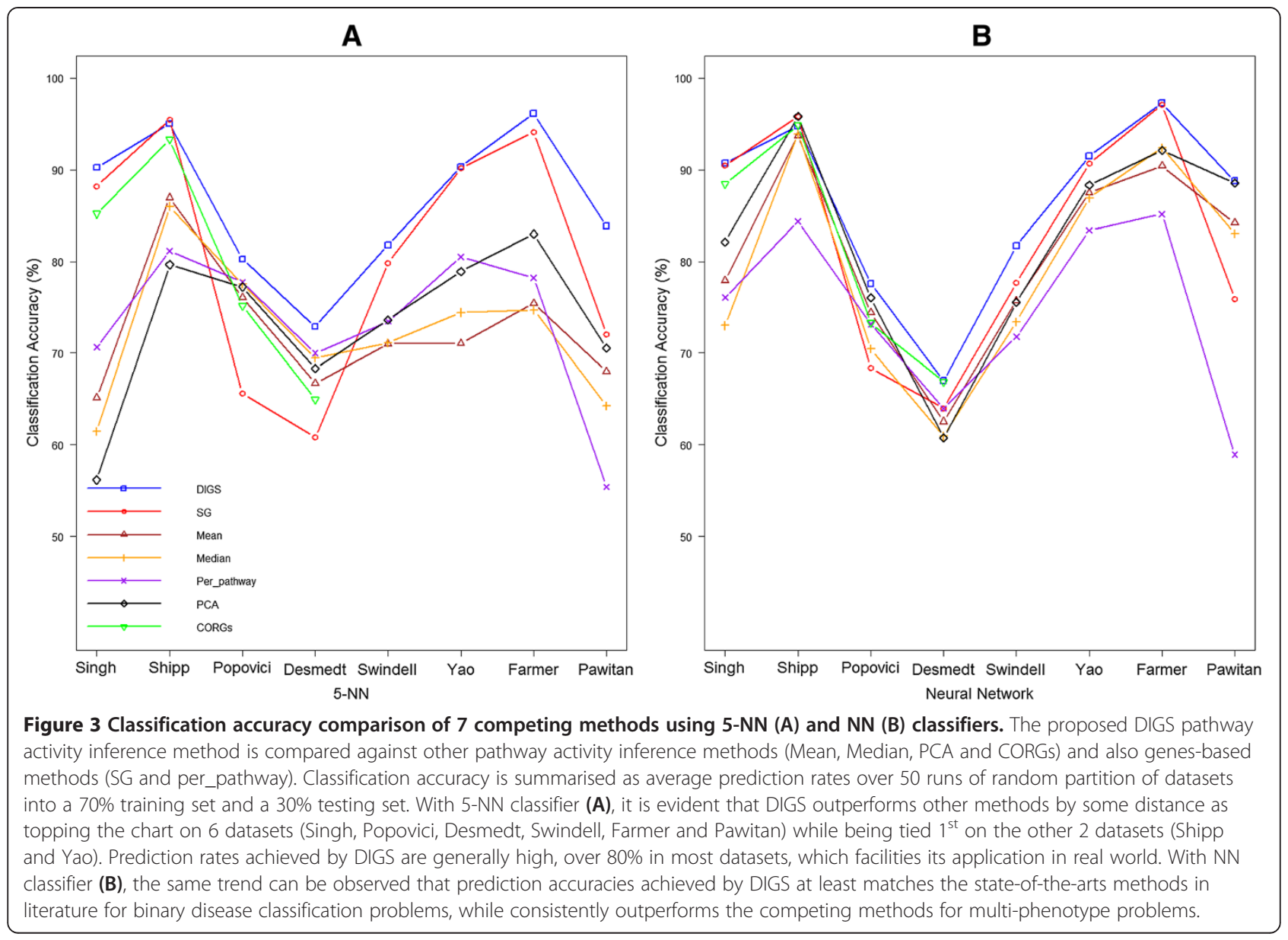

true that DIGS-based pathway activity classification approach results in higher prediction accuracy than Per_pathway, where pathway-specific gene expression profiles are trained and tested independently without constructing pathway activity features. Lastly, the same observation can be made when comparing DIGS to SG, where 186 genes of best discriminative power are selected for classification. DIGS leads to better classification rates than SG on six occasions (Singh, Popovici, Desmedt, Swindell, Farmer and Pawitan), while being tied with SG on Yao and trailing SG by marginal extent on Shipp. Overall it is evident that the proposed DIGS-based classification approach leads to more robust and accurate classification than other state-of-the-arts approaches in literature.

With regards to the actual prediction rates, the combination of DIGS model inferring pathway activity and 5 -NN classifier offers prediction rates of above $90 \%$ for 4 out of 8 employed datasets, including Singh, Shipp, Yao, Farmer, around $80 \%$ for another 3 datasets, including Popovici, Swindell and Pawitan, while still managed 70\% for the last dataset Desmedt. The generally high prediction rates demonstrate the applicability and efficiency of the proposed DIGS model in practice.
To show that the desirable prediction rates achieved by DIGS-based approach is not due to a specific bias of DIGS model with 5-NN classifier, we present the classification accuracy comparison using Neural Network classifiers in Figure 3B. According to Figure 3B, when employing Neural Network classifier, DIGS-based disease classification approach again shows great competitiveness in 4 binary datasets that it gives the highest classification rate in Popovici; is tied as the top method in Singh with single genes-based approach and in Desmedt with CORGs; in Shipp DIGS trails the most accurate approach only marginally. In terms of 4 multiclass datasets, DIGS-based classification approach dominates in all of them. The same phenomenon can be observed using the other 3 implemented classifiers that DIGS model either provides competitive classification accuracies or gives the highest classification rate (See Additional file 3 for more details).

To obtain an overview of how our methodology compares across all combinations of dataset, method and classifier, we used a simple normalisation procedure where for each pair of dataset and classifier the actual prediction rates for every method is divided by the highest prediction rates achieved throughout all methods. In other words, 
Table 3 Mean normalised classification rates over 4 two-phenotype datasets according to performance

\begin{tabular}{lllccccc}
\hline Two-class & DIGS & SG & Mean [28] & Median [28] & Per_pathway [72] & PCA [45] & CORGs [19] \\
\hline 5-NN & $\mathbf{0 . 9 9 8 8}$ & 0.9071 & 0.8737 & 0.8751 & 0.8903 & 0.8389 & 0.9371 \\
NN & $\mathbf{0 . 9 9 7 3}$ & 0.9584 & 0.9323 & 0.9004 & 0.9041 & 0.9480 & 0.9769 \\
SMO & $\mathbf{0 . 9 7 5 7}$ & 0.9474 & 0.9435 & 0.9225 & 0.9325 & 0.9704 & 0.9645 \\
HB & $\mathbf{0 . 9 8 3 5}$ & 0.9730 & 0.8819 & 0.8707 & 0.8547 & 0.8402 & 0.9595 \\
Logistic & 0.9318 & $\mathbf{0 . 9 8 1 6}$ & 0.8902 & 0.8789 & 0.8632 & 0.8482 & 0.9684 \\
Mean & $\mathbf{0 . 9 7 7 4}$ & 0.9535 & 0.9043 & 0.8895 & 0.8890 & 0.8891 & 0.9613 \\
\hline
\end{tabular}

The highest classification rate achieved across all competing methods is highlighted as bold for each classifier.

the normalised prediction rates, scaled between 0 and 1 , reflect the relative performance of a particular method compared against the best performance across all methods for this specific combination of method and classifier. For example, on the Popovici dataset with $5-\mathrm{NN}$ as classifier, the highest prediction rate across all 7 methods (achieved by DIGS as $80.14 \%$ ) is given a score of 1 and for all other methods their prediction rates are divided with the highest prediction rate (in this case for DIGS), to express the relative performance of that method to the best, e.g. raw prediction accuracy of $75.13 \%$ achieved by CORGs is normalised to: $75.13 \% / 80.14 \%=0.9375$. For each combination of method and classifier, normalised prediction rates are averaged over 4 binary datasets and 4 multiclass datasets and are shown in Tables 3 and 4 respectively.

In terms of binary datasets, Table 3 clearly indicates that DIGS pathway inference model comes at the top of all methods. This is true in the case of most classifiers used and it is only when using with logistic as classifier where DIGS is outperformed by CORGs and SG. For multi-class datasets (Table 4) DIGS is the best method throughout, indicating the strength of our proposed methodology for the most challenging cases where multiple outcomes need to be predicted. This highlights that one of the contributions of this work is to design, according to the authors' best knowledge, the first supervised pathway activity inference method applicable to both binary and multiclass datasets.

\section{DIGS release significant disease relevant pathways}

Besides the high classification rates achieved by the proposed DIGS model, we have also identified a number of breast cancer pathways that may indicate pathway biomarkers. For Pawitan, where around 90\% classification rates can be achieved using DIGS with all 5 classifiers, we employed an information gain feature ranking method in WEKA to rank the constructed pathway activities for each random training set. We record 11 pathways that are ranked more than 20 times as the most discriminative. As we have constrained the proposed DIGS model to allow only 10 genes per pathway to participant in pathway activity inference, we further extract for each identified significant pathway the set of constituent genes included in the active genes more than 10 times.

The set of pathways and genes that are found as most discriminant with our method are listed in the Table 5 below. Apart from obvious links to cancer pathways, such as prostate cancer, and other well-known signalling pathways that are known to be deregulated in tumorigenesis (Wnt signalling $[80,81]$ ), we note deregulation of nitrogen metabolism that has recently been linked to breast cancer $[82,83]$. Ubiquitin-mediated proteolysis is also identified, in accordance to previous reports about the importance of this pathway in disease [84] and is linked to poor survival in breast cancer [85]. Glycosylation is also known to be altered in cancer cells where overexpression of large glycoproteins such as mucins has been characterized [86]. Enzymes from the family of GALNT6 and GALNT14 that we have identified were found to be elevated in breast and gastric carcinomas [87]. We also identify the adherens junction complex, that comprises of cadherins and the catenins, is a major adhesion structure in endothelial cells and has been implicated in playing a fundamental role in controlling the

Table 4 Mean normalised classification rates over 4 multi-phenotype datasets according to performance

\begin{tabular}{lclcccc}
\hline Multiclass & DIGS & SG & Mean [28] & Median [28] & Per_pathway [72] & PCA [45] \\
\hline 5-NN & $\mathbf{1}$ & 0.9532 & 0.8126 & 0.8090 & 0.8158 & 0.8696 \\
NN & $\mathbf{1}$ & 0.9488 & 0.9402 & 0.9334 & 0.8322 & 0.8521 \\
SMO & $\mathbf{1}$ & 0.9335 & 0.9372 & 0.9246 & 0.7893 & 0.9452 \\
HB & $\mathbf{1}$ & 0.9241 & 0.7518 & 0.7639 & 0.5589 & 0.8043 \\
Logistic & $\mathbf{1}$ & 0.8290 & 0.5614 & 0.5440 & 0.76966 & 0.6450 \\
Mean & $\mathbf{1}$ & 0.91772 & 0.80064 & 0.79498 & 0.84452 \\
\hline
\end{tabular}

The highest classification rate achieved across all competing methods is highlighted as bold for each classifier. 
Table 5 Significant pathways and constituent genes identified by the proposed DIGS model for Pawitan

\begin{tabular}{ll}
\hline Pathway name & Significant constituent genes \\
\hline PROSTATE CANCER & EGFR, TCF7L1, GSTP1, PDGFRA, CCNE1, CHUK, PIK3R3, ERBB2, PIK3R1 \\
UBIQUITIN MEDIATED PROTEOLYSIS & UBE2E3, MID1, SKP2, BRCA1, WWP1 \\
WNT SIGNALING PATHWAY & FZD7, SOX17, TCF7L1, SKP1, SFRP1, FZD8 \\
O GLYCAN BIOSYNTHESIS & GALNT3, GALNT7, GALNT11, GALNT6, GCNT3, B4GALT5, GALNT8, C1GALT1, GALNT12, GCNT4, GALNT14, \\
& GALNT10, GALNT2, ST3GAL2, GCNT1, ST3GAL1, C1GALT1C1, GALNT1 \\
ADHERENS JUNCTION & EGFR, ERBB2, TCF7L1, TCF7L2, MET, RAC3, SMAD3, MLLT4, RHOA \\
ERBB SIGNALING PATHWAY & EGFR, NCK2, ERBB2, AKT3, PAK4, EREG, MAPK9, AKT2 \\
NITROGEN METABOLISM & CA12, CA5A, CA9, GLUL, CA3, CA14, CA8, CA7, CA5B, GLUD1, CA2, AMT, CA6, CA1, CTH, GLS2, GLUD2, \\
DORSO VENTRAL AXIS FORMATION & EGFR, NOTCH1, GRB2, MAPK3, NOTCH3, SOS1, CPEB1, PIWIL2 ETS2, MAPK1, NOTCH4, \\
ENDOMETRIAL CANCER & EGFR, TCF7L1, ERBB2, TCF7L2, MLH1, ELK1, NRAS, AKT3, ARAF, CTNNA2, PIK3CB, AKT2, CCND1, FOXO3, LEF1 \\
NON SMALL CELL LUNG CANCER & EGFR, AKT3, E2F3, ERBB2, BAD, E2F1, RARB, CDKN2A, PLCG2, GRB2, HRAS, MAPK3, PIK3CD, RXRG, TGFA \\
PANCREATIC CANCER & EGFR, ERBB2, AKT3, CDKN2A, MAPK9, PLD1, RAC3, RALA, CCND1, E2F3, JAK1, PIK3R1 \\
\hline
\end{tabular}

transport across the endothelial barrier and in regulating angiogenesis [88] and has been shown to be affected in invasive breast cancer [89].

We also draw pathway activity heat maps for the significant pathways identified in Pawitan. In Figure 4, pathway activities are inferred using all samples. Pathways are clustered based on similarity of activities on Euclidean distance.
It is clear from Figure 4 that pathways are divided into two main clusters, showing distinct patterns of expression. Ubiquitin mediated proteolysis pathway, Erbb signalling pathway, O glycan biosynthesis pathway, Dorso ventral axis formation pathway and prostate cancer pathway are shown to be associated with up-regulation in Luminal tumour, and down-regulation in Basal tumour. The other

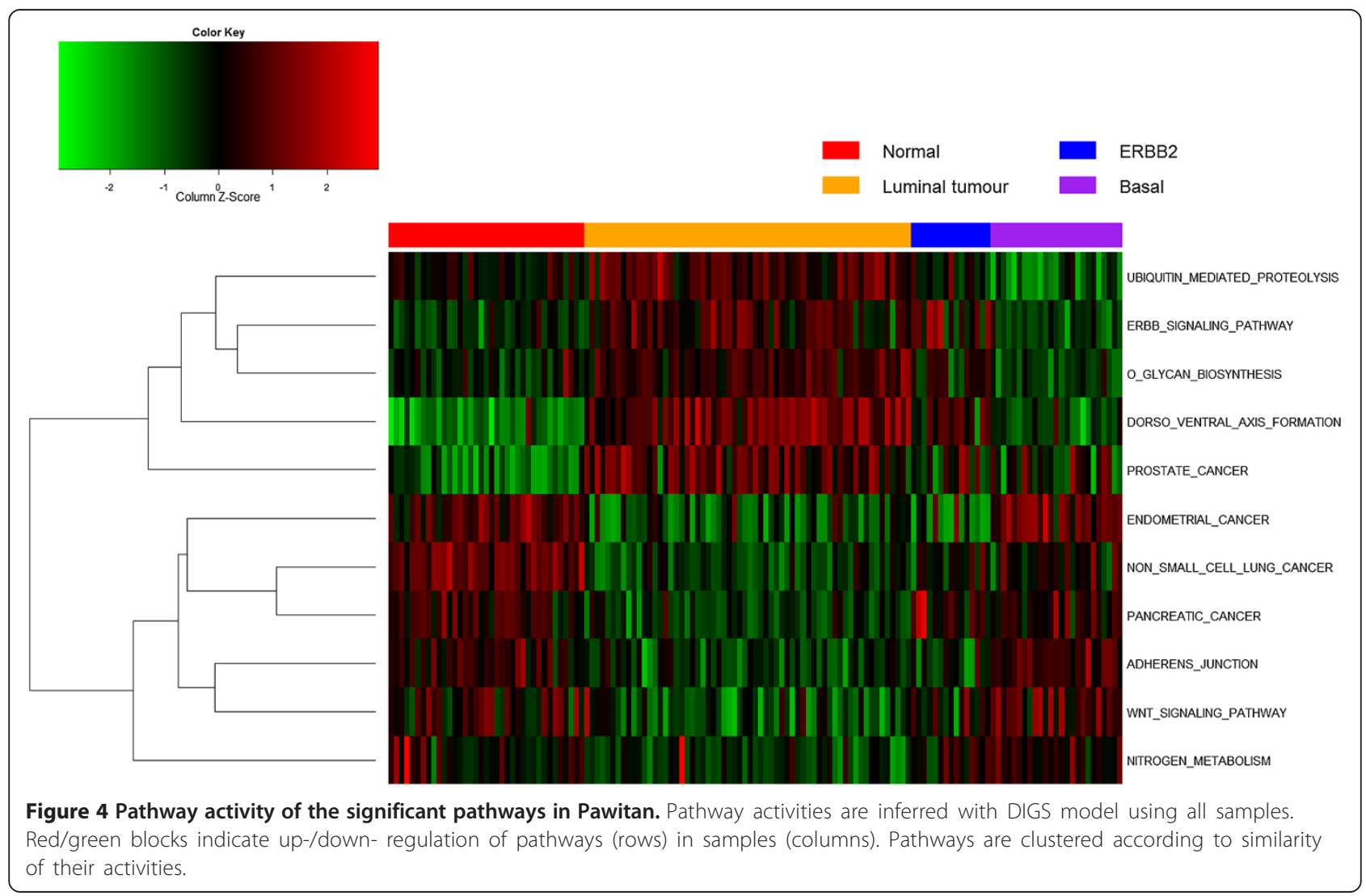


significant pathways appear to have the opposite regulation mechanism, i.e. they are down-regulated in Luminal tumour and up-regulated in Basal tumours.

We repeat the same analysis of identifying significant pathways and genes for Popovici dataset [8] and Swindell [65] where desirable classification rates can be achieved. The significant pathways/genes and heat map can be accessed at Additional files 4, 5, and 6.

\section{Conclusions}

Incorporating pathway information as biological priors into microarray gene expression profile has been demonstrated to be a promising alternative to conventional genes-based approach in various disease classification problems. However to the authors' best knowledge there are no supervised pathway activity inference methods for multiclass disease classification problems. In this work, a novel supervised pathway activity inference method for both binary and multiclass disease classification problems, DIGS, has been proposed using mathematical programming optimisation techniques. For each pathway, a new composite feature, called pathway activity, is constructed as a weighted linear summation of expressions of member genes. In each pathway the number of member genes contributing to pathway activity inference by taking non-zero weights is constrained explicitly. The proposed DIGS model provide three main benefits over the existing pathway activity inference methods in literature: (a) the weights of constituent genes in building pathway activity are optimised by DIGS in order to maximise the discriminative power of the pathway activity; (b) the maximum number of constituent genes taking non-zero weights when building pathway activity can be explicitly specified by user; (c) the proposed pathway activity inference model is applicable to both binary and multiclass disease classification problems.

A total number of 8 microarray gene expression profiles totalling 877 samples and $\sim 100,000$ genes have been used to demonstrate the applicability and efficiency of the proposed pathway activity inference scheme. The classification results show that for 4 two-class problems DIGS-based classification approaches lead to higher normalised classification performance compared to other existing pathway-based approaches as well as genesbased approaches. In terms of multiclass classification problems, mathematical programming inferring pathway activity here gives consistently the highest prediction accuracies that with the same classifier DIGS always outperforms others by distance.

\section{Additional files}

Additional file 1: Notations for mathematical model.
Additional file 2: Sensitivity analysis of parameter NoG for DIGS model with 5-NN (A), HB (B) and Logistic regression (C) classifiers. For each of the 8 datasets, the proposed DIGS model is applied to infer pathway activity while setting NoG, i.e. the maximum number of member genes in a pathway allowed to have non-zero weights, to 5, 10,15 and 20. In addition, DIGS model is also applied with NoG set to equal to the number of available member genes in a pathway, i.e. all member genes can take non-zero weights to construct pathway activity. A classifier is trained using the pathway activity profiles and tests the prediction accuracy.

Additional file 3: Classification accuracy comparison of 7 competing methods using SMO (A), HB (B) and Logistic regression (C) classifiers. The proposed DIGS pathway activity inference method is compared against other pathway activity inference methods (Mean, Median, PCA and CORGs) and also genes-based methods (SG and per_pathway). Classification accuracy is summarised as average prediction rates over 50 runs of random partition of datasets into a 70\% training set and a 30\% testing set.

Additional file 4: Significant pathways and constituent genes for Popovici dataset.

Additional file 5: Significant pathways and constituent genes for Swindell dataset.

Additional file 6: Pathway activity of the significant pathways in Swindell dataset. Pathway activities are inferred with DIGS model using all samples. Red/green blocks indicate up-/down- regulation of pathways (rows) in samples (columns). Pathways are clustered according to similarity of their activities.

\section{Competing interests}

The authors declared that they have no competing interests.

\section{Authors' contributions}

Conceived and designed the experiments: LY, LGP; Performed the experiments: LY, CA; Analysed the data: LY; Wrote the paper: LY, ST, LGP. All authors read and approved the final manusrcipt.

\section{Acknowledgements}

Funding from the UK Engineering and Physical Sciences Research Council (to LY and LGP for the EPSRC Centre for Innovative Manufacturing in Emergent Macromolecular Therapies), the UK Leverhulme Trust (to ST and LGP, RPG-2012-686) and the European Union (to ST, HEALTH-F2-2011-261366) is gratefully acknowledged.

Received: 4 July 2014 Accepted: 19 November 2014

Published online: 05 December 2014

\section{References}

1. Sorlie T, Perou CM, Tibshirani R, Aas T, Geisler S, Johnsen H, Hastie T, Eisen MB, van de Rijn M, Jeffrey SS, Thorsen T, Quist H, Matese JC, Brown PO, Botstein D, Lonning PE, Borresen-Dale AL: Gene expression patterns of breast carcinomas distinguish tumor subclasses with clinical implications. P Natl Acad Sci USA 2001, 98(19):10869-10874.

2. Khan J, Wei JS, Ringner M, Saal LH, Ladanyi M, Westermann F, Berthold F, Schwab M, Antonescu CR, Peterson C, Meltzer PS: Classification and diagnostic prediction of cancers using gene expression profiling and artificial neural networks. Nat Med 2001, 7(6):673-679.

3. Singh D, Febbo PG, Ross K, Jackson DG, Manola J, Ladd C, Tamayo P, Renshaw AA, D'Amico AV, Richie JP, Lander ES, Loda M, Kantoff PW, Golub TR, Sellers WR: Gene expression correlates of clinical prostate cancer behavior. Cancer Cell 2002, 1(2):203-209.

4. Desmedt C, Piette F, Loi S, Wang YX, D'assignies MS, Bergh J, Lidereau R, Ellis P, Harris AL, Klijn JGM, Foekens JA, Cardoso F, Piccart MJ, Buyse M, Sotiriou C: Strong time dependence of the 76-gene prognostic signature for node-negative breast cancer patients in the TRANSBIG multicenter independent validation series. Clin Cancer Res 2007, 13(11):3207-3214.

5. Wang Y, Klijn JG, Zhang Y, Sieuwerts AM, Look MP, Yang F, Talantov D, Timmermans M, Meijer-van Gelder ME, Yu J, Jatkoe T, Berns EM, Atkins D, Foekens JA: Gene-expression profiles to predict distant metastasis of lymph-node-negative primary breast cancer. Lancet 2005, 365(9460):671-679. 
6. van 't Veer $L$, Dai $H$, van de Vijver MJ, He YD, Hart AA, Mao M, Peterse HL, van der Kooy K, Marton MJ, Witteveen AT, Schreiber GJ, Kerkhoven RM, Roberts C, Linsley PS, Bernards R, Friend SH: Gene expression profiling predicts clinical outcome of breast cancer. Nature 2002, 415(6871):530-536.

7. Sotiriou C, Wirapati P, Loi S, Harris A, Fox S, Smeds J, Nordgren H, Farmer P, Praz V, Haibe-Kains B, Desmedt C, Larsimont D, Cardoso F, Peterse H, Nuyten D, Buyse M, Van de Vijver MJ, Bergh J, Piccart M, Delorenzi M: Gene expression profiling in breast cancer: understanding the molecular basis of histologic grade to improve prognosis. J Natl Cancer Inst 2006, 98(4):262-272.

8. Popovici V, Chen W, Gallas BG, Hatzis C, Shi W, Samuelson FW, Nikolsky Y, Tsyganova M, Ishkin A, Nikolskaya T, Hess KR, Valero V, Booser D, Delorenzi M, Hortobagyi GN, Shi L, Symmans WF, Pusztai L: Effect of training-sample size and classification difficulty on the accuracy of genomic predictors. Breast Cancer Res 2010, 12(1):R5.

9. Friedman DR, Weinberg JB, Barry WT, Goodman BK, Volkheimer AD, Bond KM, Chen Y, Jiang N, Moore JO, Gockerman JP, Diehl LF, Decastro CM, Potti A, Nevins JR: A genomic approach to improve prognosis and predict therapeutic response in chronic lymphocytic leukemia. Clin Cancer Res 2009, 15(22):6947-6955.

10. Peng B, Zhu D, Ander BP, Zhang X, Xue F, Sharp FR, Yang X: An integrative framework for Bayesian variable selection with informative priors for identifying genes and pathways. PLoS One 2013, 8(7):e67672.

11. Nguyen DV, Rocke DM: Tumor classification by partial least squares using microarray gene expression data. Bioinformatics 2002, 18(1):39-50.

12. Wang $S L$, Li $X L$, Fang JW: Finding minimum gene subsets with heuristic breadth-first search algorithm for robust tumor classification. BMC Bioinformatics 2012, 13:178.

13. Abeel $T$, Helleputte $T$, Van de Peer $Y$, Dupont $P$, Saeys $Y$ : Robust biomarker identification for cancer diagnosis with ensemble feature selection methods. Bioinformatics 2010, 26(3):392-398

14. Ramaswamy S, Ross KN, Lander ES, Golub TR: A molecular signature of metastasis in primary solid tumors. Nat Genet 2003, 33(1):49-54.

15. Diao HY, Li XX, Hu S, Liu YH: Gene expression profiling combined with bioinformatics analysis identify biomarkers for Parkinson disease. Plos one 2012, 7(12).

16. Schramm A, Schulte JH, Klein-Hitpass L, Havers W, Sieverts $H$, Berwanger $B$ Christiansen H, Warnat P, Brors B, Eils J, Eils R, Eggert A: Prediction of clinical outcome and biological characterization of neuroblastoma by expression profiling. Oncogene 2005, 24(53):7902-7912.

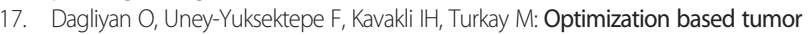
classification from microarray gene expression data. PloS one 2011, 6(2).

18. Chen $L$, Xuan JH, Riggins RB, Clarke R, Wang $Y$ : Identifying cancer biomarkers by network-constrained support vector machines. BMC Syst Biol 2011, 5:161

19. Lee E, Chuang HY, Kim JW, Ideker T, Lee D: Inferring pathway activity toward precise disease classification. Plos Comput Biol 2008, 4(11):e100021.

20. Liu W, Li CQ, Xu YJ, Yang HX, Yao QL, Han JW, Shang DS, Zhang CL, Su F. Li XX, Xiao Y, Zhang F, Dai M, Li X: Topologically inferring risk-active pathways toward precise cancer classification by directed random walk. Bioinformatics 2013, 29(17):2169-2177.

21. Rapaport F, Zinovyev A, Dutreix M, Barillot E, Vert JP: Classification of microarray data using gene networks. BMC Bioinformatics 2007, 8:35.

22. Ein-Dor L, Kela I, Getz G, Givol D, Domany E: Outcome signature genes in breast cancer: is there a unique set? Bioinformatics 2005, 21(2):171-178.

23. Zeng LY, Yu J, Huang T, Jia HL, Dong QZ, He F, Yuan WL, Qin LX, Li YX, $X i e$ L: Differential combinatorial regulatory network analysis related to venous metastasis of hepatocellular carcinoma. BMC Genomics 2012 13(Suppl 8):S14

24. Chuang HY, Lee E, Liu YT, Lee D, Ideker T: Network-based classification of breast cancer metastasis. Mol Syst Biol 2007, 3:140

25. Su JJ, Yoon BJ, Dougherty ER: Accurate and reliable cancer classification based on probabilistic inference of pathway activity. PloS one 2009, 4(12).

26. Drier $Y$, Sheffer $M$, Domany E: Pathway-based personalized analysis of cancer. P Natl Acad Sci USA 2013, 110(16):6388-6393.

27. Yang RT, Daigle BJ, Petzold LR, Doyle FJ: Core module biomarker identification with network exploration for breast cancer metastasis. BMC Bioinformatics 2012, 13:12.

28. Guo Z, Zhang TW, Li X, Wang Q, Xu JZ, Yu H, Zhu J, Wang HY, Wang CG, Topol EJ, Wang Q, Rao SQ: Towards precise classification of cancers based on robust gene functional expression profiles. BMC Bioinformatics 2005, 6:58.
29. Kim YA, Wuchty S, Przytycka TM: Identifying causal genes and dysregulated pathways in complex diseases. Plos Comput Biol 2011, 7(3):e1001095.

30. Chuang HY, Rassenti L, Salcedo M, Licon K, Kohlmann A, Haferlach T, Foa R, Ideker T, Kipps TJ: Subnetwork-based analysis of chronic lymphocytic leukemia identifies pathways that associate with disease progression. Blood 2012, 120(13):2639-2649.

31. Wu MY, Dai DQ, Zhang XF, Zhu Y: Cancer subtype discovery and biomarker identification via a New robust network clustering algorithm. Plos one 2013, 8(6):e66256.

32. Su J, Yoon BJ, Dougherty ER: Identification of diagnostic subnetwork markers for cancer in human protein-protein interaction network. BMC Bioinformatics 2010, 11(Suppl 6):S8.

33. Nibbe RK, Markowitz S, Myeroff L, Ewing R, Chance MR: Discovery and scoring of protein interaction subnetworks discriminative of late stage human colon cancer. Mol Cell Proteomics 2009, 8(4):827-845.

34. Azuaje F: What does systems biology mean for biomarker discovery? Expert Opin Med Diagn 2010, 4(1):1-10.

35. Kanehisa M, Goto S, Kawashima S, Okuno Y, Hattori M: The KEGG resource for deciphering the genome. Nucleic Acids Res 2004, 32:D277-D280.

36. Ashburner M, Ball CA, Blake JA, Botstein D, Butler H, Cherry JM, Davis AP, Dolinski K, Dwight SS, Eppig JT, Harris MA, Hill DP, Issel-Tarver L, Kasarskis A, Lewis S, Matese JC, Richardson JE, Ringwald M, Rubin GM, Sherlock G, Consortium GO: Gene ontology: tool for the unification of biology. Nat Genet 2000, 25(1):25-29.

37. Joshi-Tope G, Gillespie M, Vastrik I, D'Eustachio P, Schmidt E, de Bono B, Jassal B, Gopinath GR, Wu GR, Matthews L, Lewis S, Birney E, Stein L: Reactome: a knowledgebase of biological pathways. Nucleic Acids Res 2005, 33:D428-D432.

38. Subramanian A, Tamayo P, Mootha VK, Mukherjee S, Ebert BL, Gillette MA, Paulovich A, Pomeroy SL, Golub TR, Lander ES, Mesirov JP: Gene set enrichment analysis: a knowledge-based approach for interpreting genome-wide expression profiles. P Natl Acad Sci USA 2005, 102(43):15545-15550.

39. Huang DW, Sherman BT, Tan Q, Collins JR, Alvord WG, Roayaei J, Stephens R, Baseler MW, Lane HC, Lempicki RA: The DAVID gene functional classification tool: a novel biological module-centric algorithm to functionally analyze large gene lists. Genome Biol 2007, 8(9):R183.

40. Al-Shahrour F, Arbiza L, Dopazo H, Huerta-Cepas J, Minguez P, Montaner D, Dopazo J: From genes to functional classes in the study of biological systems. BMC Bioinformatics 2007, 8:114.

41. Glaab E, Schneider R: PathVar: analysis of gene and protein expression variance in cellular pathways using microarray data. Bioinformatics 2012, 28(3):446-447

42. Hanzelmann S, Castelo R, Guinney J: GSVA: gene set variation analysis for microarray and RNA-Seq data. BMC Bioinformatics 2013, 14:7.

43. Tian L, Greenberg SA, Kong SW, Altschuler J, Kohane IS, Park PJ: Discovering statistically significant pathways in expression profiling studies. $P$ Nat Acad Sci USA 2005, 102(38):13544-13549.

44. Ackermann M, Strimmer K: A general modular framework for gene set enrichment analysis. BMC Bioinformatics 2009, 10:47.

45. Bild AH, Yao G, Chang JT, Wang QL, Potti A, Chasse D, Joshi MB, Harpole D, Lancaster JM, Berchuck A, Olson JA, Marks JR, Dressman HK, West M, Nevins JR: Oncogenic pathway signatures in human cancers as a guide to targeted therapies. Nature 2006, 439(7074):353-357.

46. Tamayo P, Cho YJ, Tsherniak A, Greulich H, Ambrogio L, Schouten-van Meeteren N, Zhou TN, Buxton A, Kool M, Meyerson M, Pomeroy SL, Mesirov JP: Predicting relapse in patients with medulloblastoma by integrating evidence from clinical and genomic features. J Clin Oncol 2011, 29(11):1415-1423.

47. Vaske CJ, Benz SC, Sanborn JZ, Earl D, Szeto C, Zhu JC, Haussler D, Stuart JM: Inference of patient-specific pathway activities from multi-dimensional cancer genomics data using PARADIGM. Bioinformatics 2010, 26(12):i237-i245.

48. Liu KQ, Liu ZP, Hao JK, Chen LN, Zhao XM: Identifying dysregulated pathways in cancers from pathway interaction networks. BMC Bioinformatics 2012, 13:126.

49. Chen $X$, Wang LL: Integrating biological knowledge with gene expression profiles for survival prediction of cancer. J Comput Bio/ 2009, 16(2):265-278.

50. Jiao Y, Lawler K, Patel GS, Purushotham A, Jones AF, Grigoriadis A, Tutt A, $\mathrm{Ng} \mathrm{T}$, Teschendorff AE: DART: Denoising Algorithm based on Relevance network Topology improves molecular pathway activity inference. BMC Bioinformatics 2011, 12:403. 
51. Yang XA, Regan K, Huang Y, Zhang QB, Li JR, Seiwert TY, Cohen EEW, Xing HR, Lussier YA: Single sample expression-anchored mechanisms predict survival in head and neck cancer. Plos Comput Biol 2012, 8(1):e1002350.

52. Staiger C, Cadot S, Kooter R, Dittrich M, Muller T, Klau GW, Wessels LFA: A critical evaluation of network and pathway-based classifiers for outcome prediction in breast cancer. PloS one 2012, 7(4):e34796.

53. Perera GK, Ainali C, Semenova E, Hundhausen C, Barinaga G, Kassen D, Williams AE, Mirza MM, Balazs M, Wang X, Rodriguez RS, Alendar A, Barker J, Tsoka S, Ouyang W, Nestle FO: Integrative biology approach identifies cytokine targeting strategies for psoriasis. Sci Transl Med 2014, 6(223):223ra222.

54. Iorio MV, Ferracin M, Liu CG, Veronese A, Spizzo R, Sabbioni S, Magri E, Pedriali M, Fabbri M, Campiglio M, Menard S, Palazzo JP, Rosenberg A, Musiani P, Volinia S, Nenci I, Calin GA, Querzoli P, Negrini M, Croce CM: MicroRNA gene expression deregulation in human breast cancer. Cancer Res 2005, 65(16):7065-7070.

55. Kleer CG, Cao Q, Varambally S, Shen RL, Ota L, Tomlins SA, Ghosh D, Sewalt RGAB, Otte AP, Hayes DF, Sabel MS, Livant D, Weiss SJ, Rubin MA, Chinnaiyan AM: EZH2 is a marker of aggressive breast cancer and promotes neoplastic transformation of breast epithelial cells. P Natl Acad Sci USA 2003, 100(20):11606-11611.

56. Sotiriou C, Neo SY, MCShane LM, Korn EL, Long PM, Jazaeri A, Martiat $P$, Fox SB, Harris AL, Liu ET: Breast cancer classification and prognosis based on gene expression profiles from a population-based study. $P$ Natl Acad Sci USA 2003, 100(18):10393-10398.

57. Carey LA, Perou CM, Livasy CA, Dressler LG, Cowan D, Conway K, Karaca G, Troester MA, Tse CK, Edmiston S, Deming SL, Geradts J, Cheang MCU, Nielsen TO, Moorman PG, Earp HS, Millikan RC: Race, breast cancer subtypes, and survival in the Carolina breast cancer study. Jama-J Am Med Assoc 2006, 295(21):2492-2502

58. Tsoi LC, Spain SL, Knight J, Ellinghaus E, Stuart PE, Capon F, Ding J, Li YM, Tejasvi T, Gudjonsson JE, Kang HM, Allen MH, McManus R, Novelli G, Samuelsson L, Schalkwijk J, Stahle M, Burden AD, Smith CH, Cork MJ, Estivill X, Bowcock AM, Krueger GG, Weger W, Worthington J, Tazi-Ahnini R, Nestle FO, Hayday A, Hoffmann P, Winkelmann J, et al: Identification of 15 new psoriasis susceptibility loci highlights the role of innate immunity. Nat Genet 2012, 44(12):1341-1348.

59. Nestle FO, Kaplan D, Barker J: Psoriasis. New Engl J Med 2009, 361(17):1710-1710

60. Ainali C, Valeyev N, Perera G, Williams A, Gudjonsson J, Ouzounis C, Nestle F, Tsoka S: Transcriptome classification reveals molecular subtypes in psoriasis. BMC Genomics 2012, 13(1):472.

61. Dhanasekaran SM, Barrette TR, Ghosh D, Shah R, Varambally S, Kurachi K, Pienta KJ, Rubin MA, Chinnaiyan AM: Delineation of prognostic biomarkers in prostate cancer. Nature 2001, 412(6849):822-826.

62. Lapointe J, Li C, Higgins JP, van de Rijn M, Bair E, Montgomery K, Ferrari M, Egevad L, Rayford W, Bergerheim U, Ekman P, DeMarzo AM, Tibshirani R, Botstein D, Brown PO, Brooks JD, Pollack JR: Gene expression profiling identifies clinically relevant subtypes of prostate cancer. P Natl Acad Sci USA 2004, 101(3):811-816.

63. Shipp MA, Ross KN, Tamayo P, Weng AP, Kutok JL, Aguiar RCT, Gaasenbeek M, Angelo M, Reich M, Pinkus GS, Ray TS, Koval MA, Last KW, Norton A Lister TA, Mesirov J, Neuberg DS, Lander ES, Aster JC, Golub TR: Diffuse large B-cell lymphoma outcome prediction by gene-expression profiling and supervised machine learning. Nat Med 2002, 8(1):68-74.

64. Alizadeh AA, Eisen MB, Davis RE, Ma C, Lossos IS, Rosenwald A, Boldrick JG, Sabet H, Tran T, Yu X, Powell JI, Yang LM, Marti GE, Moore T, Hudson J, Lu LS, Lewis DB, Tibshirani R, Sherlock G, Chan WC, Greiner TC, Weisenburger DD, Armitage JO, Warnke R, Levy R, Wilson W, Grever MR, Byrd JC, Botstein D, Brown PO, et al: Distinct types of diffuse large B-cell lymphoma identified by gene expression profiling. Nature 2000, 403(6769):503-511.

65. Swindell WR, Johnston A, Carbajal S, Han GW, Wohn C, Lu J, Xing XY, Nair RP, Voorhees JJ, Elder JT, Wang XJ, Sano S, Prens EP, DiGiovanni J, Pittelkow MR, Ward NL, Gudjonsson JE: Genome-wide expression profiling of five mouse models identifies similarities and differences with human psoriasis. PloS one 2011, 6(4):e18266.

66. Yao YH, Richman L, Morehouse C, Delos Reyes M, Higgs BW, Boutrin A White B, Coyle A, Krueger J, Kiener PA, Jallal B: Type I interferon: potential therapeutic target for psoriasis? PloS one 2008, 3(7):e2737.

67. Farmer $P$, Bonnefoi $H$, Becette $V$, Tubiana-Hulin $M$, Fumoleau $P$, Larsimont $D$, MacGrogan G, Bergh J, Cameron D, Goldstein D, Duss S, Nicoulaz AL, Brisken
C, Fiche M, Delorenzi M, lggo R: Identification of molecular apocrine breast tumours by microarray analysis. Oncogene 2005, 24(29):4660-4671.

68. Pawitan Y, Bjohle J, Amler L, Borg AL, Egyhazi S, Hall P, Han X, Holmberg L, Huang F, Klaar S, Liu ET, Miller L, Nordgren H, Ploner A, Sandelin K, Shaw PM, Smeds J, Skoog L, Wedren S, Bergh J: Gene expression profiling spares early breast cancer patients from adjuvant therapy: derived and validated in two population-based cohorts. Breast Cancer Res 2005, 7(6):R953-R964.

69. Smyth GK: limma: Linear Models for Microarray Data. In Bioinformatics and Computational Biology Solutions Using R and Bioconductor. Edited by Gentleman R, Carey V, Huber W, Irizarry R, Dudoit S. New York: Springer; 2005:397-420.

70. Liberzon A, Subramanian A, Pinchback R, Thorvaldsdottir H, Tamayo $P$, Mesirov JP: Molecular signatures database (MSigDB) 3.0. Bioinformatics 2011, 27(12):1739-1740.

71. lannarilli FJ, Rubin PA: Feature selection for multiclass discrimination via mixed-integer linear programming. leee T Pattern Anal 2003, 25(6):779-783.

72. Ainali C, Nestle F, Papageorgiou LG, Tsoka S: Disease classification through integer optimisation. Comput-Aided Chem En 2011, 29:1548-1552.

73. Keerthi SS, Shevade SK, Bhattacharyya C, Murthy KRK: Improvements to Platt's SMO algorithm for SVM classifier design. Neural Comput 2001, 13(3):637-649.

74. Rumelhart DE, Hinton GE, Williams RJ: Learning Internal Representations by Error Propagation. In Parallel Distributed Processing: Explorations in the Microstructure of Cognition, Volume 1. Edited by David ER, James LM. Cambridge, MA, USA: Group CPR: MIT Press; 1986:318-362.

75. Aha DW, Kibler D, Albert MK: Instance-based learning algorithms. Mach Learn 1991, 6(1):37-66.

76. Cessie L, van Houwelingen JC: Ridge estimators in logistic regression. Applied Statistics 1992, 41(1):191-201.

77. Xu G, Papageorgiou LG: A mixed integer optimisation model for data classification. Comput Ind Eng 2009, 56(4):1205-1215.

78. GAMS Development Corporation: General Algebraic Modeling System (GAMS) Release 24.2.1. Washington, DC, USA: 2013.

79. Hall M, Frank E, Holmes G, Pfahringer B, Reutemann P, Witten IH: The WEKA data mining software: an update. SIGKDD Explor News/ 2009, 11(1):10-18.

80. Howe LR, Brown AMC: Wnt signaling and breast cancer. Cancer Biol Ther 2004, 3(1):36-41

81. Lamb R, Ablett MP, Spence K, Landberg G, Sims AH, Clarke RB: Wnt pathway activity in breast cancer Sub-types and stem-like cells. PloS one 2013, 8(7):e67811.

82. Bernhardt S, Mitra D, Soons Z, Konig R, Vetter M, Thomssen C, Kantelhardt E, Wiemann S, Korf U: Profiling metabolic changes in breast cancer with targeted proteomics. Cancer Metab 2014, 2(Suppl 1):7.

83. Mitra D, Bernhardt S, Soons Z, Poschet G, Hell R, Koenig R, Korf U, Wiemann $S:$ Metabolic transformations in breast cancer subtypes. Cancer Metab 2014, 2(Suppl 1):48.

84. Ohta T, Fukuda M: Ubiquitin and breast cancer. Oncogene 2004, 23(11):2079-2088.

85. Cepeda D, Ng HF, Sharifi HR, Mahmoudi S, Cerrato VS, Fredlund E, Magnusson K, Nilsson H, Malyukova A, Rantala J, Klevebring D, Vinals F, Bhaskaran N, Zakaria SM, Rahmanto AS, Grotegut S, Nielsen ML, Szigyarto CAK, Sun DH, Lerner M, Navani S, Widschwendter M, Uhlen M, Jirstrom K, Ponten F, Wohlschlegel J, Grander D, Spruck C, Larsson LG, Sangfelt O: CDK-mediated activation of the SCFFBXO28 ubiquitin ligase promotes MYC-driven transcription and tumourigenesis and predicts poor survival in breast cancer. Embo Mol Med 2013, 5(7):1067-1086.

86. Taylor-Papadimitriou J, Burchell JM, Miles D, Sewell R: Changes in mucin-type O-glycosylation in breast cancer: implications for the host immune response. Int J Exp Pathol 2004, 85(4):A52-A52.

87. Meany D, Cha D: Aberrant glycosylation associated with enzymes as cancer biomarkers. Clin Proteom 2011, 8(1):1-14.

88. Iyer S, Ferreri DM, DeCocco NC, Minnear FL, Vincent PA: VE-cadherin-p120 interaction is required for maintenance of endothelial barrier function. Am J Physiol-Lung C 2004, 286(6):L1143-L1153.

89. Haidari M, Zhang W, Wakame K: Disruption of endothelial adherens junction by invasive breast cancer cells is mediated by reactive oxygen species and is attenuated by AHCC. Life Sci 2013, 93(25-26):994-1003.

doi:10.1186/s12859-014-0390-2

Cite this article as: Yang et al:: Pathway activity inference for multiclass disease classification through a mathematical programming optimisation framework. BMC Bioinformatics 2014 15:390. 